Radiologe

1998·38: 972-986 ○ Springer-Verlag 1998

Redaktion

S. Feuerbach • Regensburg

G. van Kaick $\cdot$ Heidelberg

K.G. Hering $\cdot$ Dortmund

M.Walz $\cdot$ Mannheim

Die Beiträge der Rubrik,"Weiterbildung“ sollen dem Stand des zur Facharztprüfung für den Arzt für Diagnostische Radiologie notwendigen Wissens entsprechen und zugleich dem niedergelassenen Facharzt als Repetitorium dienen. Die Rubrik beschränkt sich auf klinisch gesicherte Aussagen zum Thema.

\section{Diagnostische Möglichkeiten}

Auffinden der Tubusspitze auf dem Röntgenbild
A. Bankier • D. Fleischmann • L. Aram ${ }^{1} \cdot$ K. Heimberger • E. Schindler • C. J. Herold • Universitätsklinik für Radiodiagnostik, Wien, ${ }^{1}$ Universitätsklinik für Anästhesie und Allgemeine Intensivmedizin, Wien

\section{Bildgebung in der Intensivmedizin}

\section{Techniken, Indikationen, diagnostische Zeichen - Teil I}

\begin{abstract}
Die rasant fortschreitenden Entwicklungen auf dem Gebiet der Radiodiagnostik eröffnen auch der Intensivmedizin neue Aspekte: Mittels moderner Technik können heute ungleich rascher konklusive Diagnosen gestellt werden. Dies setzt jedoch die Kenntnis der Möglichkeiten und auch der Grenzen bildgebender Methoden in der Intensivmedizin voraus. Im vorliegenden Beitrag werden, ausgehend von relevanten klinischen Fragestellungen und Problemkomplexen, die Möglichkeiten bildgebender Verfahren vorgestellt und diskutiert. Es werden die wichtigsten diagnostischen Röntgenzeichen erläutert und ein diagnostisches Prozedere vorgezeichnet. Somit soll der vorliegende Beitrag eine kurze Zusammenfassung der für die Intensivmedizin wichtigen Aspekte der Bildgebung darstellen.
\end{abstract}

e bildgebende Diagnostik hat in den letzten beiden Jahrzehnten eine durch technische Weiterentwicklungen bedingte rasante Entwicklung erfahren. Auf die Bildgebung in der Intensivmedizin hat dies insofern Auswirkungen, als sich durch die zum Teil völlig neuartigen Methoden nunmehr $\boldsymbol{~ d i a g n o s t i s c h e ~ M o ̈ g l i c h k e i t e n ~}$ bieten, die in dieser Form für die Intensivmedizin bislang nicht verfügbar waren. Dadurch hat sich auch die Rolle des Radiologen in der Intensivmedizin neu definiert. Neben dem rein diagnostischen Prozedere hat dieser nunmehr auch die Funktion, ein anhand des gegebenen klinischen Kontextes adäquates diagnostisches Vorgehen mit zu definieren. Im vorliegenden Artikel werden neben den Basiszeichen der intensivmedizinisch-radiologischen Diagnostik anhand klinisch relevanter Fragestellungen auch die Einsatzmöglichkeiten moderner bildgebender Diagnostik besprochen werden, die den nunmehr verfügbaren neuen Methoden den ihnen entsprechenden Stellenwert zuweisen.

\section{Radiologisches Monitoring des beatmeten Patienten}

\section{Endotrachealer Tubus}

Der „handelsübliche“ endotracheale Tubus trägt eine linienförmige röntgendichte Markierung, die das Auffinden der Tubusspitze auf dem Röntgenbild erleichtert. Diese Spitze sollte - bei neutraler Position des Patientenkopfes - idealerweise 5 bis 7 $\mathrm{cm}$ kranial der Karina liegen. Bei überstrecktem Hals gleitet die Tubusspitze um bis

Ao. Univ.-Prof. Dr. A. Bankier • Universitätsklinik für Radiodiagnostik, Währinger Gürtel 18-20, A-1090 Wien 


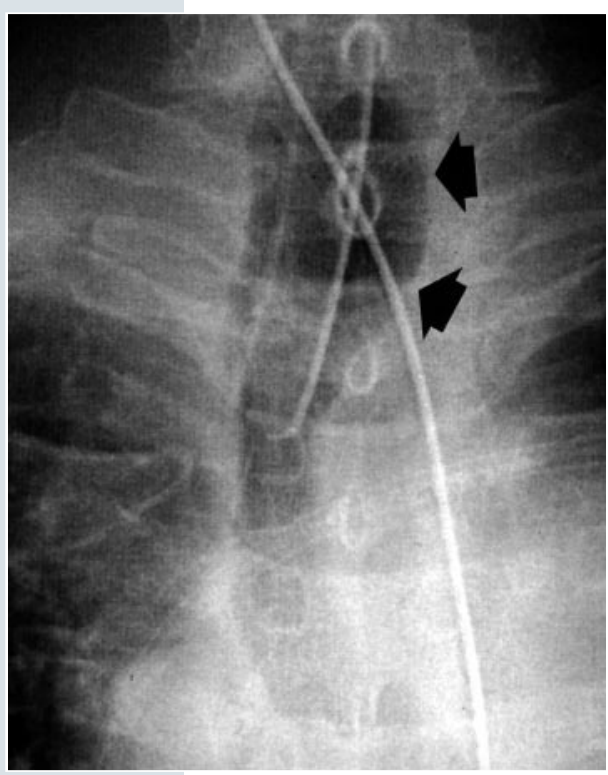

Endotrachealer Tubus. Diese Vergrößerung aus einer digitalen Thoraxaufnahme zeigt eine im Verhältnis zur Karina zu hoch positionierte Tubusspitze und einen deutlich überblähten Tubus-Cuff (Pfeile)

Die Kopfposition des Patienten sollte bei der Bildanfertigung bekannt sein.

\section{Weite der Trachea}

Digitales Thoraxbild

Ermöglicht die Visualisierung schlecht sichtbarer Tubusspitzen

Komplikationen der endotrachealen Intubation

Das IE ist eine relativ häufige Komplikation der Überdruckbeatmung.

Radiologische Erkennung des IE

Transparente Streifen Erste Zeichen des IE sind hilifugale, periphere Aufhellungsstreifen. zu $3 \mathrm{~cm}$ nach kranial, bei gebeugtem Hals um die gleiche Distanz nach kaudal. Dies erklärt, weshalb bei neutraler Kopfposition die Tubusspitze der Karina nicht näher als $5 \mathrm{~cm}$ liegen sollte: Bei einer kürzeren Distanz könnte nämlich die alleinige Änderung der Kopfposition zur einseitigen endobronchialen Fehlintubation führen. Deshalb sollte bei der radiologischen Lagebeurteilung des Tubus die Kopfposition des Patienten zum Zeitpunkt der Bildanfertigung bekannt sein.

Die Weite der Trachea in Höhe des insufflierten Cuffs sollte den Durchmesser des Tubus nicht wesentlich, keinesfalls aber um mehr als das Doppelte überschreiten (Abb. 1). Dies ist deshalb von Bedeutung, da überblähte Cuffs schon nach einigen Minuten zu Durchblutungsstörungen der Trachealschleimhaut und in weiterer Folge zu potentiell irreparablen Schleimhautschädigungen führen können. Im allgemeinen ist das suffiziente radiologische Monitoring des endotrachealen Tubus mittels Thoraxröntgen in einer Ebene möglich.

Das digitale Thoraxbild bietet gegenüber dem analogen Thoraxbild die Möglichkeit, mittels elektronischer Bildverarbeitung Tubusspitzen zu visualisieren, die durch Überlagerung mit anatomischen Strukturen oder mit anderen Zugängen schlecht sichtbar sind. Mögliche Komplikationen der endotrachealen Intubation bestehen in der Fehlintubation des rechten Hauptbronchus mit konsekutiver Atelektase der linken Lunge, in ungenügendem Vorschieben des Tubus mit konsekutiver Schädigung der Larynxstrukturen, in der tracheobronchialen Obstruktion durch Verlegung des Tubuslumens mit Schleim oder Blut, und, bei langzeitintubierten Patienten, in der Trachealruptur durch längerfristig überblähte Cuffs mit konsekutivem Luftaustritt ins Mediastinum, ins Perikard und in die Pleuralhöhle [7,18].

\section{Interstitielles Emphysem (IE)}

Während das Vorliegen von Luft im Interstitium bei Neugeborenen mit Syndrom der hyalinen Membranen eine bekannte Komplikation des Barotraumas darstellt, hat das interstitielle Emphysem (IE) des Erwachsenen erst während der letzten 15-20 Jahre im Zuge der zunehmenden Verbreitung der Überdruckbeatmung weiterreichende Aufmerksamkeit erlangt.

Die radiologische Erkennung des IE erfordert neben Röntgenbildern von optimaler Qualität die genaue anatomische Kenntnis der thorakalen Bindegewebsräume. Das früheste radiologisch erkennbare Zeichen eines IE ist das plötzliche Auftreten von transparenten Streifen, die in ungeordneter Weise von den Hili zur Lungenperipherie ziehen. Anders als Luftbronchogramme zeigen diese Streifen keine Aufzweigungen und vermindern ihr Kaliber auch in peripherer Lage nicht. 


\section{Halos \\ Peripheres Interstitium}

Zystische Luftansammlungen Bei Fortschreiten des IEs.

\section{Pneumothorax \\ Cave: Aus dem IE kann sich ein \\ Pneumothorax entwickeln!}

Prognose

Eindringen freier Luft
Die anatomische Entsprechung dieser transparenten Streifen ist Luft im sogenannten „axialen Interstitium“, d.h. im peribronchovaskulären Bindegewebe. Ist ein Gefäß, dessen peribronchovaskuläres Bindegewebe luftgefüllt ist, tangential getroffen, kommt es zur Ausbildung von charakteristischen ringförmigen perivaskulären Aufhellungen, den sogenannten „Halos“. Zum vorwiegend streifigen Aspekt des IE tragen vornehmlich luftgefüllte interlobuläre Septen ( „peripheres Interstitium“) bei. Die zufällige Anordnung der sekundären Lobuli bedingt den unorganisierten Aspekt dieser Veränderungen, die am einfachsten in subpleuraler Lokalisation zu erkennen sind. Sie erscheinen dann radiologisch wie das „Negativbild“ der im Kapitel „Lungenödem“ beschriebenen Kerley-Linien.

Bei Fortschreiten des IEs bilden sich $>$ zystische Luftansammlungen mit Durchmessern im Millimeterbereich aus, die jedoch nur dann gut sichtbar sind, wenn sie unmittelbar subpleural liegen. Durchsetzen diese zystischen Luftbläschen die Lunge generalisiert und diffus, bleibt eine diffuse Transparenzerhöhung des Thorax deren einziges radiologisches Zeichen. Da die subpleuralen zystenartigen Luftaufhellungen in subpleuraler Lage durch das Vorliegen von Luft im peripheren Interstitium bedingt sind, müssen sie, insbesonders im beatmeten Patienten, als Warnzeichen vor einem drohenden $>$ Pneumothorax gewertet werden. Während zentral gelegene emphysembedingte Pneumatozelen im beatmeten Patienten, unabhängig von ihrer Größe, nur selten klinische Komplikationen hervorrufen, sollten selbst diskrete Zeichen eines peripheren IEs immer ernstgenommen und insbesonders bei der Planung des Beatmungsregimes berücksichtigt werden.

Das Ausmaß und die Ausdehnung des IEs lassen keine direkten Rückschlüsse auf dessen Prognose zu. So können diskrete IEs schnell zu gravierenden Komplikationen führen, andererseits aber können ausgedehnte IEs über Wochen hin radiologisch unverändert bleiben.

\section{Pneumothorax}

Aufgrund des im Pleuraraum herrschenden Unterdrucks und der darin ausgespannten Lunge führt das Eindringen von freier Luft durch die Eigenelastizität des Lungenparenchyms zum partiellen oder totalen Kollaps des betroffenen Lun-
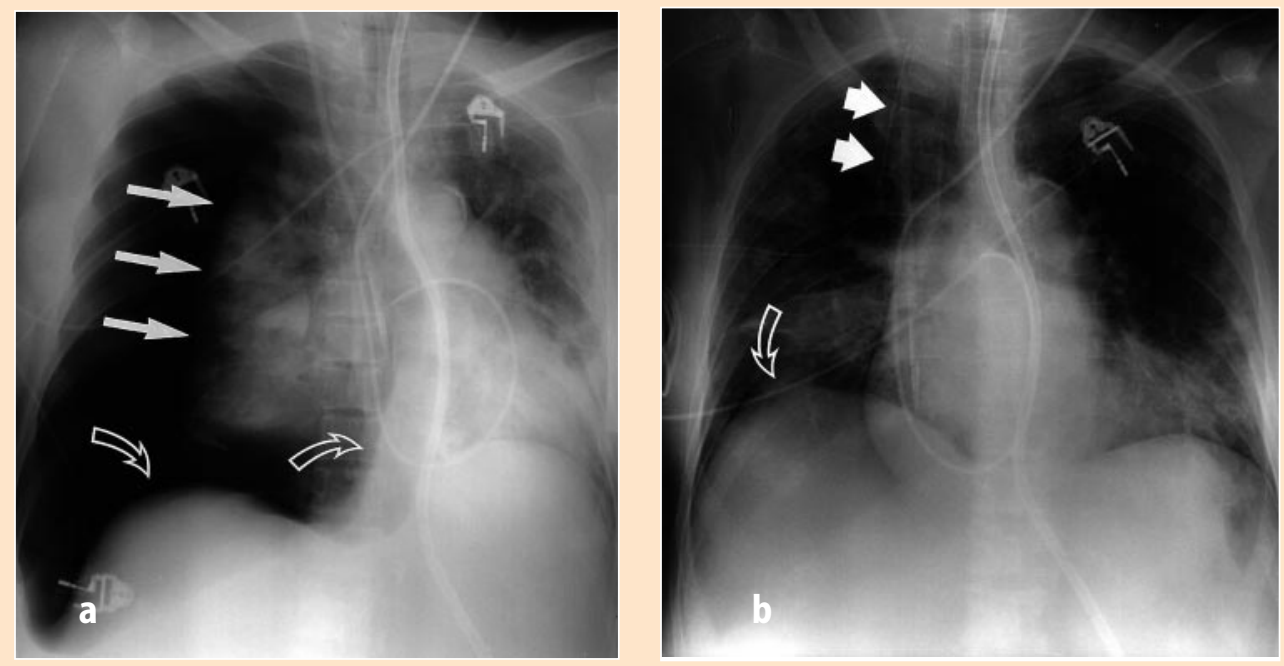

Abb. 2 А

Pneumothorax. Die beiden Aufnahmen zeigen einen hochgradigen Spannungs-Pneumothorax vor (a) und nach (b) Pleuradrainage: b zeigt sowohl den ausgedehnten Pneuspalt (gerade Pfeile) als auch die Zwerchfelldepression und die Mediastinalverschiebung zur Gegenseite (gebogene Pfeile); b zeigt beide Veränderungen nach Insertion eines Pleuradrains (gerade Pfeile) rückgebildet. Das Zwerchfell (gebogener Pfeil) ist wieder in annähernd normaler Lage, ein Pneuspalt ist nicht mehr nachweisbar 


\section{Pleurale Verletzung \\ Pneuspalt \\ Klassisches Zeichen eines Pneumothorax. physem bzw. Mediastinalemphysem erreichen oder durch Verletzung der Pleura von außen eindringen. Diese pleurale Verletzung kann traumatischer (z. B. An- spießung durch frakturierte Rippe) oder iatrogener (z. B. Anstechen bei zentraler Venenpunktion) Genese sein. \\ Das klassische radiologische Zeichen eines Pneumothorax ist der sogenannte - „Pneuspalt“, d.h. die feine, von der Lungenoberfläche hervorgerufene Linie, wel- che nach distal hin ein luftgefülltes Areal begrenzt, in dem keinerlei Lungenstruktu- ren mehr aufzufinden sind. Beim stehenden Patienten sammelt sich aufgrund der Schwerkraft freie pleurale Luft meist im Bereich der Lungenspitzen. Im Regelfall er- scheint dann der Pneumothorax umso augenscheinlicher, je größer dessen Ausdeh- nung ist. Voraussetzung hierfür bleibt allerdings, daß die Lungenoberfläche von den Röntgenstrahlen tangential getroffen wird und so eine klar erkennbare Grenzfläche bildet (Abb. 2,3).}

genflügels. Luft kann den Pleuralraum über ein vorbestehendes interstitielles Em-

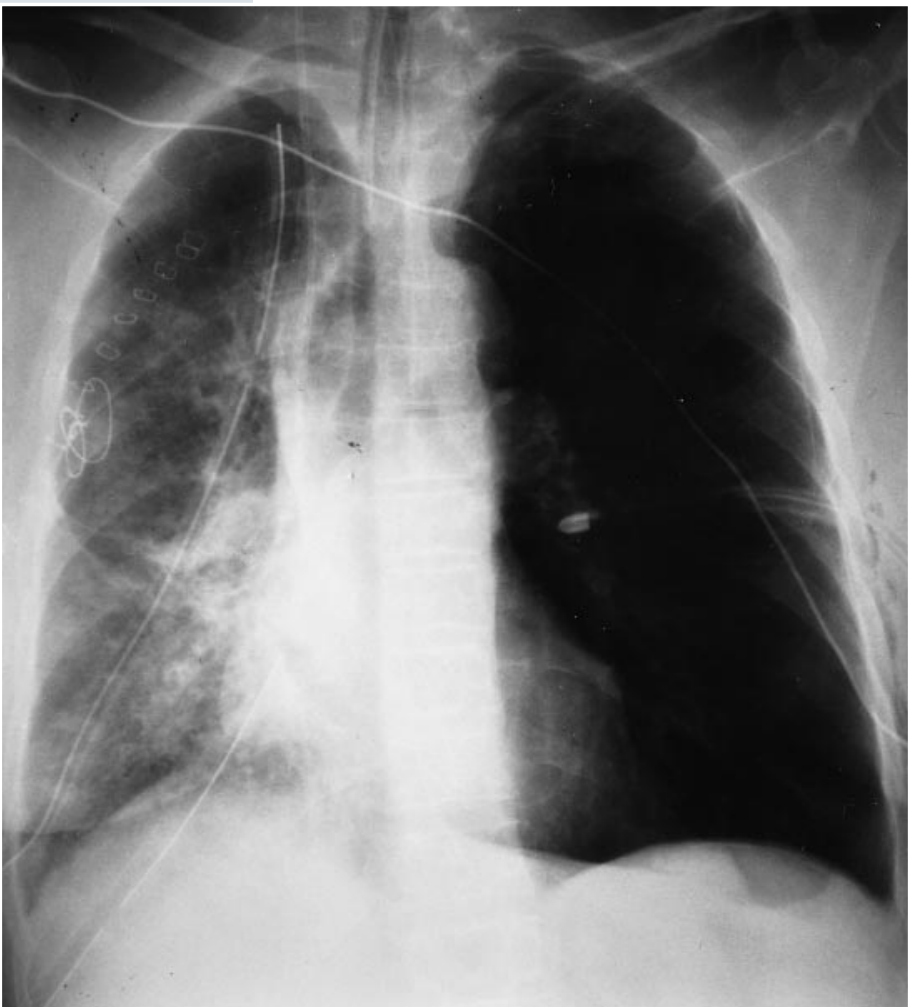

Abb 3

Thoraxröntgen bei einem Patienten nach Lungentransplantation. Während die transplantierte rechte Lunge noch nicht vollständig entfaltet ist, zeigt sich links ein schmaler lateraler Pneumothorax sowie ein geringes laterales Weichteilemphysem, zu dessen Entstehen auch das lateral eingebrachte Pleuradrain beigetragen haben dürfte

\section{Radiologische Darstellung eines Pneumothorax}

Indirekte Röntgenzeichen

Zusätzliche Röntgenaufnahmen:

- Quertisch-Seitaufnahme

- Tangentialaufnahme
Die radiologische Darstellung eines Pneumothorax beim Intensivpatienten bereitet aus bereits erwähnten Gründen spezifische Schwierigkeiten [4, 17]: Aufgrund der liegenden Patientenposition sammelt sich die freie pleurale Luft nicht im Bereich der Lungenspitzen, sondern meist ventral bzw. subpulmonal. Da meist nur eine Aufnahme im antero-posterioren Strahlengang vorliegt, gelingt es oft nicht, einen „Pneuspalt“ einwandfrei darzustellen. Zudem ist die Lunge des beatmeten Patienten bei intakter viszeraler Pleura trotz lädierter parietaler Pleura relativ gut entfaltet, sodaß der Pneuspalt insgesamt klein bleibt.

Daher erfordert der Nachweis eines Pneumothorax im Intensivpatienten neben der Suche nach indirekten Röntgenzeichen möglicherweise die Anfertigung zusätzlicher Röntgenaufnahmen. Ziel dieser zusätzlichen Aufnahmen ist die tangentiale Darstellung der Oberfläche der kollabierten Lunge. Hierfür kann sowohl eine Quertisch-Seitaufnahme als auch eine Tangentialaufnahme angefertigt werden. In den Händen eines geübten Untersuchers geben diese Aufnahmen genü- 


\section{Optische Demarkierung thorakaler Grenzflächen}

Indirekte Röntgenzeichen den Pneumothorax. gend Informationen, um das Vorliegen einen Pneumothorax zu bestätigen oder auszuschließen. Der routinemäßige Einsatz der Computertomographie allein zur Diagnose des Pneumothorax sollte Ausnahmefällen vorbehalten bleiben.

Wie oben erwähnt, sammelt sich freie pleurale Luft im liegenden Intensivpatienten meist ventral oder subpulmonal. Dadurch kommt es zu ungewöhnlich deutlicher optischer Demarkierung von thorakalen Grenzflächen, wie sie im stehenden Patienten mit Pneumothorax nur selten gesehen werden. Diese Grenzflächendemarkierungen gelten als indirekte Röntgenzeichen des Pneumothorax. Die genaue Lokalisation der Grenzflächendemarkierungen bzw. die betroffenen anatomischen Strukturen sind von Seite und Lage der pleuralen Luftansammlung abhängig. Die Tabellen 1 und 2 fassen die wichtigsten indirekten Zeichen des Pneumothorax zusammen.

Tabelle 1

Die wichtigsten radiologischen Zeichen des anteromedialen Pneumothorax

D Suprahilärer anteromedialer Pneumothorax

Scharfe Demarkierung von:

Vena cava superior

Vena azygos

Linke Arteria subclavia

Vordere Pleuraumschlagfalte

Obere Pulmonalvene

Infrahilärer anteromedialer Pneumothorax

Scharfe Demarkierung von:

Herzrand

Vena cava inferior

Tiefer anteriorer kardiophrenischer Sulkus

Mediales Zwerchfell unter der Herzsilhouette

Scharfe Demarkierung des perikardialen Fettbürzels

Tabelle 2

Die wichtigsten radiologischen Zeichen des subpulmonalen Pneumothorax

Dypertransparente obere Quadranten

D Tiefe kostophrenische Sulci

- Scharfe diaphragmale Begrenzung

- Sichtbarwerdung der anterioren und posterioren diaphragmalen Sulci

Dichtbarwerdung der Vena cava inferior

\section{Pneumomediastinum}

Luft im Mediastinum ist bei Intensivpatienten meist die Folge eines Barotraumas, doch können auch andere Veränderungen wie z.B. eine Ruptur im Bereich der Luftwege oder des Ösophagus zur Ausbildung eines Pneumomediastinums führen. Auch ein interstitielles Emphysem kann entlang der bronchovaskulären Bündel bis ins Mediastinum disseziieren (Abb.4).

Radiologisch ist das Pneumomediastinum daran erkennbar, daß es durch Luftaufhellungen anatomische Grenzflächen von Mediastinalstrukturen sichtbar macht, die für gewöhnlich nicht sichtbar sind. Besonders von diesem Phänomen betroffen sind der mediale Rand der Vena cava superior, der linken Arteria subclavia, der linken Arteria carotis communis und der rechten Vena inominata. Auch die Visualisierung der Vena azygos oder der Vena intercostalis superior kann als sicheres Röntgenzeichen des Pneumomediastinums gelten. Außerdem kann mediastinale 
Luft auch um die Pulmonalarterien und um die Aorta aszendens radiologisch sichtbar werden. Weil die beiden Seiten des Mediastinums in Verbindung stehen, kann mediastinale Luft beide zentralen Anteile des Zwerchfells unter der Herzsilhouette demarkieren und unterscheidet sich in dieser Fähigkeit prinzipiell vom subpulmonalen Pneumothorax. Die anatomische Kontinuität zwischen Mediastinum und Retroperitoneum ermöglicht es zudies, daß freie mediastinale Luft ins Retroperitoneum übertritt.

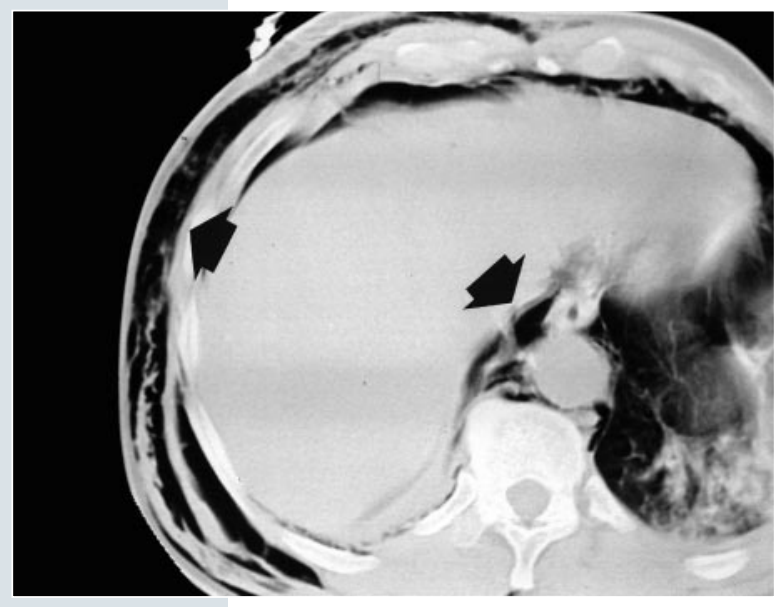
Diese CT-Schicht in Höhe des thorako-abdominellen Übergangs zeigt sowohl ein ausgeprägtes Weichteilemphysem als auch eine deutliche Luftansammlung zwischen Zwerchfell, Aorta und Ösophagus (schwarze Pfeile). Der Patient hat ein ausgeprägtes Barotrauma erlitten

Pulmonale Prozesse

Radiologische Verdichtungen als Folge.

Atemdruck: entscheidendes Merkmal.

Die o.g. Veränderungen sind vollständig reversibel.

Cave: Bei beatmeten Intensivpatienten sind Änderungen im Beatmungsregime von großer Bedeutung!

\section{PEEP}

Mechanische Beatmung, im besonderen PEEP (positive endexpiratory pressure), kann zu maßgeblichen Änderungen sowohl des Thoraxröntgens als auch des Thorax-CTs führen. Da erhöhter Beatmungsdruck zu einer vermehrt luftgefüllten Lunge führt, können pathologische Prozesse ihr radiologisches Bild auf artifizielle Art ändern, obwohl sie selbst nicht von dieser Änderung betroffen sind. Dies gilt für alle pulmonalen Prozesse, die zu radiologischen Verdichtungen führen, insbesonders für das Lungenödem, jedoch auch für das ARDS sowie für Pneumonien. Als Faustregel kann hier gelten: wird der Atemdruck in einem Patienten mit gleichbleibend ausgedehnter Verdichtung erhöht, so scheint diese Verdichtung nach Erhöhung des Atemdrucks radiologisch weniger ausgedehnt und/oder weniger dicht. Die Lunge wirkt besser transparent, die Gefäßzeichnung erscheint schlanker und schärfer.

Der zugrundeliegende physiologische Mechanismus ist hierbei, daß Flüssigkeit aus den Alveolen durch den erhöhten Atmungsdruck ins Lungenbindegewebe „hinausgepreßt" wird. Auch kann, liegt keine intraalveoläre Flüssigkeit vor, zusätzlich durch den relativ größeren Luftgehalt der Lunge die Abnahme einer Verdichtung vorgetäuscht werden. Diese Veränderungen sind vollständig rückbildungsfähig: bei Rücknahme des Beatmungsdrucks stellt sich der ursprüngliche radiologische $\mathrm{Zu}$ stand der Verdichtung wieder her.

In CT-Studien konnte gezeigt werden, daß ein erhöhter Atmungsdruck bei Intensivpatienten mit atelektatischen Lungenarealen $\mathrm{zu}$ einer teilweisen Wiederbelüftung dieser Areale führen kann. Demgegenüber bleibt das schon suffizient belüftete Lungengewebe unverändert. Bei Intensivpatienten mit atelektatischen Veränderungen wird die klinische und radiologische Verbesserung also durch teilweise Wiedergewinnung ehemals atelektatischer Lungenbezirke bewirkt und nicht durch Überventilation von noch gesundem Lungengewebe.

Aus oben genannten Gründen ist es im Fall von beatmeten Intensivpatienten von großer Bedeutung, über eventuell stattgehabte Änderungen im Beatmungsregime zu erfahren. Anderenfalls kann dies zu Interpretationsirrtümern führen. 


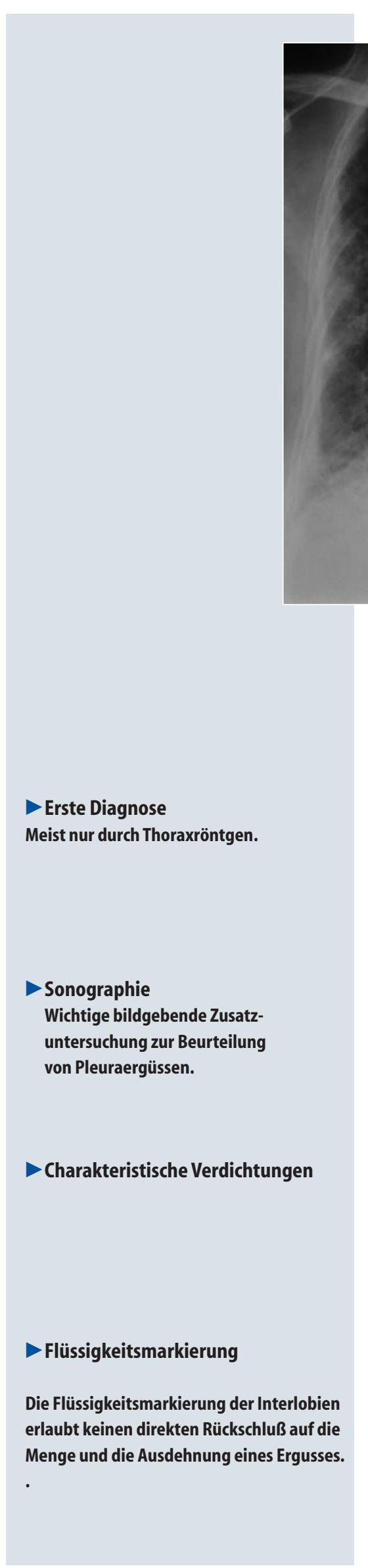

\section{Freie pleurale Flüssigkeit}

Freie pleurale Flüssigkeit in Form von pleuralen Ergüssen ist bei intensivpflichtigen Patienten häufig, bleibt jedoch, insbesonders bei mengenmäßig wenig ausgeprägten Ergüssen, schwierig zu diagnostizieren, da zur $\$ ersten Diagnose meist nur das Thoraxbettröntgen zur Verfügung steht. Wiewohl in der Computertomographie auch im liegenden Patienten selbst kleine Ergüsse zweifelsfrei nachgewiesen werden können, bedarf es dieser aufwendigen Untersuchung zur alleinigen Diagnose eines Pleuraergusses meist nicht, da selbst ausgedehntere Ergüsse erst bei klinisch instabilen Patienten zu funktionell relevanten Einschränkungen der Atemmechanik führen. Als wichtige bildgebende Zusatzuntersuchung zur Beurteilung von Pleuraergüssen hat sich in den letzten Jahren die Sonographie erwiesen: durch sie kann ohne Patientenbelastung und direkt am Krankenbett durch Nachweis der charakteristischen echofreien Lamelle im Pleuraraum das Vorliegen eines Ergusses bestätigt werden.

Da sich größere pleurale Flüssigkeitsmengen der Schwerkraft folgend verteilen, kommt es sowohl in halbsitzenden Patienten als auch in Kopftieflage zu charakteristischen basalen bzw. apikalen kappenförmigen oder meniskusartigen homogenen - Verdichtungen, die sich, bei intakter Pleura und fehlendem Pneumothorax, entlang der lateralen Thoraxwandanteile verschmälern und an Dichte abnehmen [19] (Abb.5). Im flach liegenden Patienten kann eine diskrete homogene Verdichtung eines Hemithorax der einzige radiologische Hinweis für das Vorliegen eines Pleuraergusses sein; dieses Zeichen ist jedoch diskret und oftmals sind Vorbilder nötig, um es von einer durch einen Rastereffekt verursachten Pseudoverdichtung zu differenzieren.

Auch die Flüssigkeitsmarkierung der pleuralen Interlobien kann wichtige radiologische Hinweise auf das Vorliegen eines Pleuraergusses geben. Die Flüssigkeitsmarkierung der Interlobien kann jedoch nicht als direkter Rückschluß auf die Menge und die Ausdehnung eines Ergusses verwendet werden, da sie in hohem Maß davon abhängt, wie die betreffenden Interlobien tangential im Strahlengang liegen und ob es zusätzliche pleurale Veränderungen (Synechien, inkomplette Fissuren) gibt, die das radiologische Aussehen des Ergusses beeinflussen können.

Zusammenfassend sollte neben dem Thoraxröntgen, welches im Zweifelsfall kurzfristig mit anderer Patientenlagerung (z.B. Seitlage) zu wiederholen ist, die So- 
Pleuradrainage

Die Lage des Pleuradrains ist entscheidend.

Bei Verdacht auf Drainagefehllagen sollten Thoraxaufnahmen in zwei Ebenen durchgeführt werden.

Extrathorakale Fehllagen

Computertomographie Zur weiteren Abklärung einer suspizierten Drainfehllage.

Zahlreiche Faktoren bedingen die hohe Pneumonie-Inzidenz.

Radiologischer Hinweise auf Erreger

Lungenröntgen

\section{Thoraxröntgen}

Pulmonale Parenchymverdichtung nographie eine zunehmende Rolle bei der Evaluierung pleuraler Ergüsse im intensivpflichtigen Patienten erhalten, da sie wenig belastend, schnell und zuverlässig über Vorhandensein, Verteilung und ungefähre Menge des Ergusses Auskunft geben kann [18].

Die Therapie der Wahl im Falle größerer oder funktionell wirksamer Pleuraergüsse stellt die Pleuradrainage dar. Hierbei sollte die Lage des Pleuradrains radiologisch monitiert werden, da der alleinige Verlaß auf das Funktionieren des Drains irreführend sein kann. So kann zum Beispiel ein extrathorakal liegender Drain, der eine Interkostalarterie verletzt hat, durchaus hämorragische Flüssigkeit fördern und somit eine regelrechte Lage vortäuschen. Die Drainspitze projiziert sich radiologisch idealerweise auf den punctum maximum der zu drainierenden Flüssigkeitsansammlung. Allgemeingültige radiologische Kriterien für eine regelrechte Drainlage gibt es nicht, jedenfalls sollte das Drain auf dem a.p.-Bild eine leichte Kurve nach oben oder nach unten beschreiben, weil dies auf einen regelrechten intrapleuralen Drainverlauf hinweist [15,18]. Schon im Zuge der ersten radiologischen Kontrolle kann eine Aufnahme in einer zweiten Ebene angestrebt werden, eine solche sollte aber spätesten bei Auftreten klinischer Komplikationen angefertigt werden, da zahlreiche Drain-Fehllagen in einer Ebene nur schwer zu erkennen sind.

$\mathrm{Zu}$ den möglichen Komplikationen pleuraler Drainagen zählen $>$ extrathorakale Fehllagen mit konsekutivem Weichteilemphysem, intrafissurale Fehllagen und intrapulmonale Fehllagen mit konsekutivem intraparenchymalen Hämatom. Im Falle inkonklusiver konventioneller Thoraxaufnahmen kann die Computertomographie zur weiteren Abklärung einer suspizierten Drainfehllage dienen. Sie ermöglicht es, überlagerungsfrei das topographische Verhältnis des Drains zu umgebenden pulmonalen oder mediastinalen Strukturen darzustellen.

\section{Radiologische Fokussuche in der Thoraxbildgebung}

\section{Thorakaler Fokus, Pneumonie}

Die hohe Pneumonie-Inzidenz auf Intensivstationen wird durch zahlreiche Faktoren verursacht. Grunderkrankungen wie Diabetes, Malignome, chronische Atemwegserkrankungen oder hämatologische Erkrankungen können das Entstehen von Pneumonien begünstigen. Lange Hospitalisierungen führen zu Veränderungen der Pharynxflora in Richtung eines Spektrums, das reich an Gram-negativen Organismen ist. Längerdauernde Antibiotikatherapie oder die Verabreichung von Steroiden begünstigen eine rasche Kolonialisierung.

Physiologische Barrieren können durch den Einsatz von Endotrachealtuben und zentralvenösen Kathetern umgangen werden. So ist bei den meisten über mehrere Tage intubierten Patienten eine Keim-Kolonisierung der Trachea nachweisbar. Schließlich führen zahlreiche Medikamente zu einer $\mathrm{pH}$-Änderung im Magen, welche wiederum vermehrtes gastrisches Keimwachstum hervorruft. Wird dieser keimreiche Mageninhalt aspiriert, wirkt er als zusätzliche endogene Infektionsquelle.

Die ideale radiologische Klassifikation der Pneumonien entspräche einer Einteilung nach Erregern. Leider sind viele Pneumonie-assoziierte Veränderungen weder im Lungenröntgen noch im CT so spezifisch, daß sie einen sicheren Rückschluß auf einen bestimmten Erreger zulassen. Dieser Umstand ist im Rahmen der Intensivstation umso gravierender, als hier andere Veränderungen wie z.B. verschiedene Formen des Lungenödems Pneumonie-ähnliche radiographische Bilder hervorbringen können. Trotzdem hat die Bildgebung bei Intensivpatienten mit suspizierten Pneumonien große Bedeutung: Das $>$ Lungenröntgen ist als bildgebende Methode die erste Wahl, die sowohl das Vorliegen einer Pneumonie objektivieren als auch deren Lokalisation und Ausdehnung bestimmen kann. Prädisponierende Faktoren (Tubusfehllagen, maligne Raumforderungen, Aspiration) können ebenso sichtbar gemacht werden wie eventuelle Komplikationen (Pleuraergüsse, Empyeme, Abszesse). Schließlich bietet das Thoraxröntgen die Möglichkeit, nach der Erstdiagnose der Pneumonie den post-therapeutischen Verlauf zu monitieren. Das wesentliche radiographische Merkmal der Pneumonie ist die $>$ pulmonale Parenchymverdichtung. Das Spektrum dieser Verdichtungen kann von einer solitären 

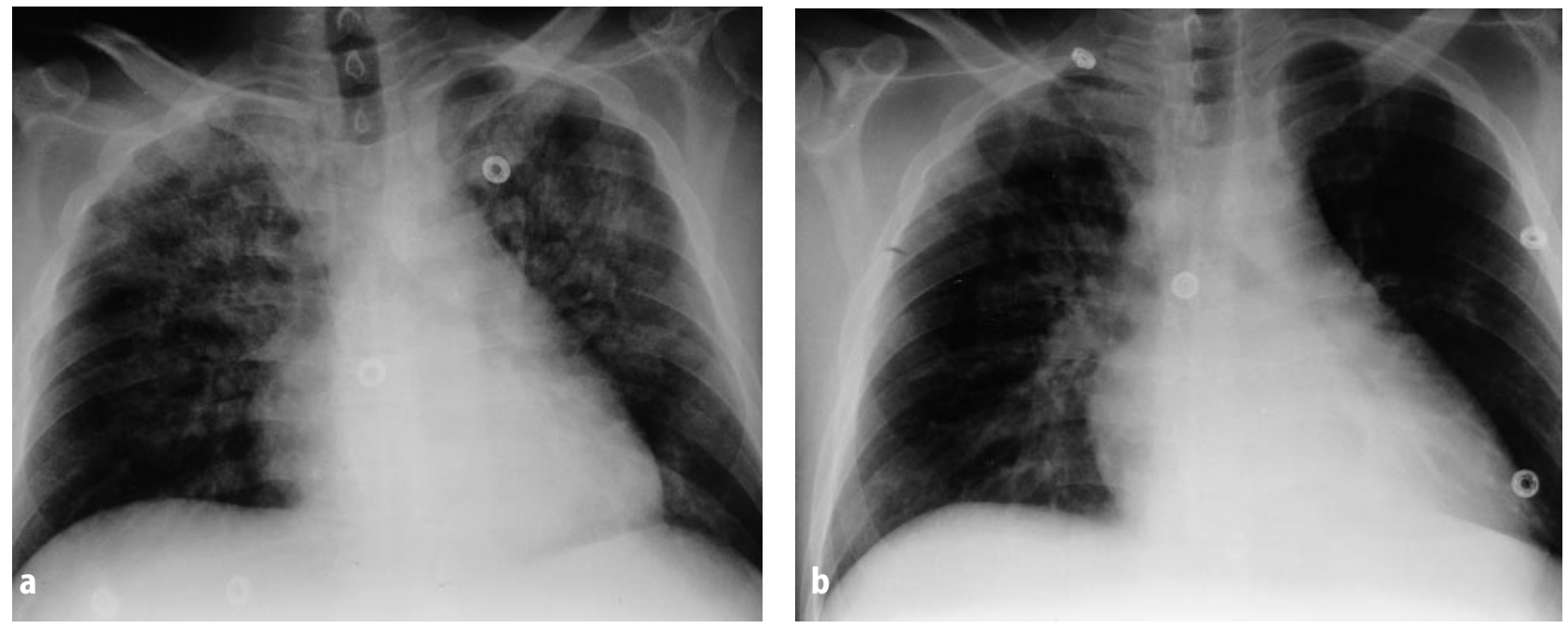

\section{Abb.6 $\Delta$}

Bakteriell bedingte Oberlappenpneumonie in einem bereits extubierten Intensivpatienten. Das Bild zeigt teils retikuläre, teils noduläre Verdichtungen mit vereinzelten Luftbronchogrammen (a).

Nach acht Tagen antiobiotischer Therapie sind die Verdichtungen praktisch vollständig rückgebildet (b)

\section{Unterschiedliche \\ Verdichtungsformen}

Die Suche nach dem möglichen Erreger sollte im Vordergrund jeder klinischen Diagnostik stehen.

\section{Unilaterale, segmentale oder lobäre Verdichtungen}

Meist bakteieller Genese.

\section{Diffuse bilaterale Verdichtungen Ursache sind oft Viren und Protozoen.}

\section{Verdichtungen mit Kavitationen Vor allem bei Pilz- oder bakteriellen Pneumonien.}

diskreten Dichteerhöhung bis zur ausgeprägten Konsolidierung einer ganzen Lunge reichen.

Unabhängig von ihrer Ausdehnung werden die pulmonalen Verdichtungen nach ihrer Morphologie in noduläre, miliare, streifige, netzartige und fleckförmige Verdichtungen unterteilt. Das Vorliegen dieser unterschiedlichen Verdichtungsformen hängt nicht nur vom jeweiligen Erreger, sondern auch vom Immunstatus des Patienten ab. Aufgrund ihres radiographischen Bildes werden Pneumonien auch in Bronchopneumonien, Lobärpneumonien und interstitielle Pneumonien unterteilt. Obwohl diese Begriffe häufig angewendet werden, haben sie nur begrenzte praktische Bedeutung, da die gleichen Erreger unterschiedliche Verdichtungsbilder hervorrufen können und verschiedene Verdichtungsbilder bei ein und demselben Patienten überlappen können.

Weil neben der eigentlichen Objektivierung einer suspizierten Pneumonie die Suche nach dem möglichen Erreger im Vordergrund jeder klinischen Diagnostik steht, soll im folgenden anhand stichwortartig zusammengefaßter Merkpunkte gezeigt werden, in welcher Weise die radiologische Diagnostik die klinische Diagnostik ergänzen kann bzw. welche klinischen Gesichtspunkte in die Interpretation von Intensiv-Thoraxbildern einfließen sollten.

Streng unilaterale, segmentale oder lobäre Verdichtungen, wie sie bei nichtvorerkrankten Patienten angetroffen werden, sind bei Intensivpatienten sehr selten und dann zumeist von Bakterien verursacht (Abb.6). Die wichtigsten Differentialdiagnosen sind hierbei das lokalisierte Lungenödem und das einseitige ARDS. - Diffuse bilaterale Verdichtungen sind meist durch Viren oder Protozoen verursacht (Abb. 7,8$)$

Bei lokalisierten Veränderungen sollte in jedem Fall an das Vorliegen einer postobstruktiven Pneumonie gedacht werden und eine mögliche Obstruktion (Schleim, Fremdkörper, Fehlintubation, intrabronchiale Raumforderung) bronchoskopisch ausgeschlossen werden. Großflächige Konsolidierungen können durch Klebsiellen, S. aureus, Legionellen oder Gram-negative Keime hervorgerufen werden. Die Volumenerhöhung eines konsolidierten Lungenareals legt das Vorliegen von Pneumokokken oder Klebsiellen nahe.

- Verdichtungen mit Kavitationen machen bakterielle oder Pilzpneumonien wahrscheinlicher als Virus- oder Mykoplasmen-Infektionen, wobei zu beachten ist, daß Pilzpneumonien am ehesten bei Immunsuprimierten auftreten. Bakterien, die am häufigsten Kavitationen hervorrufen, sind S. aureus, Klebsiellen, Proteus, Pseudomonas, Anaerobier und M. tuberculosis. Eine große solitäre homogene Verdichtung 

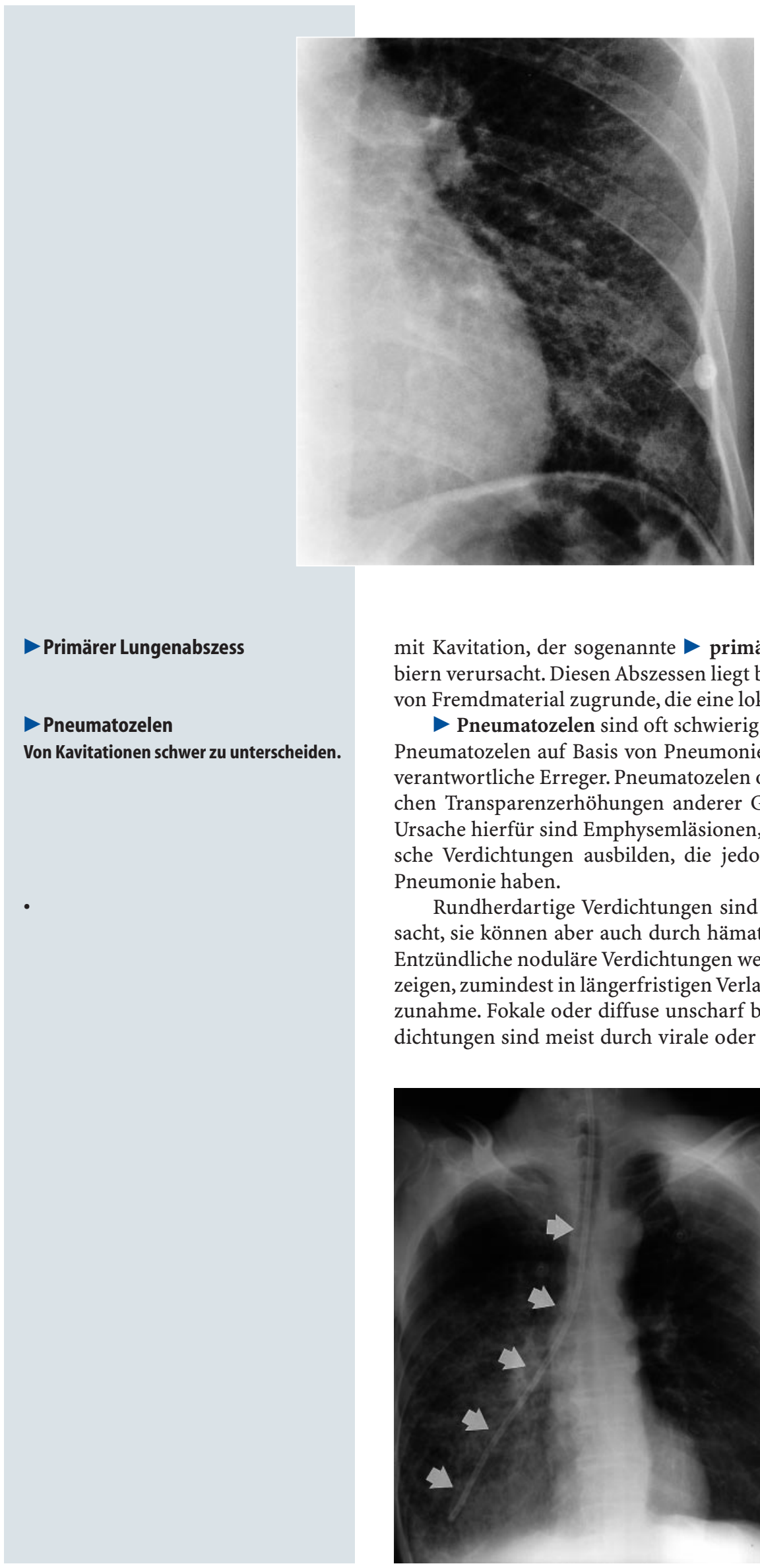

4 Abb. 7 Lungenröntgen (Detail) eines Intensivpatienten. Das Bild zeigt diffuse retikunoduläre Verdichtungen, die, im Gegensatz zu Abb. 6, keine Luftbronchogramme aufweisen. Eine Infektion des Lungenparenchyms mit dem Cytomegalie-Virus wurde bioptisch gesichert

mit Kavitation, der sogenannte primäre Lungenabszess, ist meist von Anaerobiern verursacht. Diesen Abszessen liegt bei Intensivpatienten häufig die Aspiration von Fremdmaterial zugrunde, die eine lokale Abwehrstörung verursacht.

- Pneumatozelen sind oft schwierig von Kavitationen zu unterscheiden. Wenn Pneumatozelen auf Basis von Pneumonien entstehen, ist meist S. aureus der dafür verantwortliche Erreger. Pneumatozelen oder Kavitationen sollten nicht mit rundlichen Transparenzerhöhungen anderer Genese verwechselt werden. Die häufigste Ursache hierfür sind Emphysemläsionen, in deren lokalem Umfeld sich pneumonische Verdichtungen ausbilden, die jedoch keinen kausalen Zusammenhang zur Pneumonie haben.

Rundherdartige Verdichtungen sind meist durch Pilze oder Nocardien verursacht, sie können aber auch durch hämatogene Streuung von Bakterien entstehen. Entzündliche noduläre Verdichtungen weisen oftmals Luftbronchogramme auf und zeigen, zumindest in längerfristigen Verlaufskontrollen über mehrere Tage, Größenzunahme. Fokale oder diffuse unscharf begrenzte bilaterale retikulo-noduläre Verdichtungen sind meist durch virale oder mykoplasmen-bedingte Pneumonien ver-

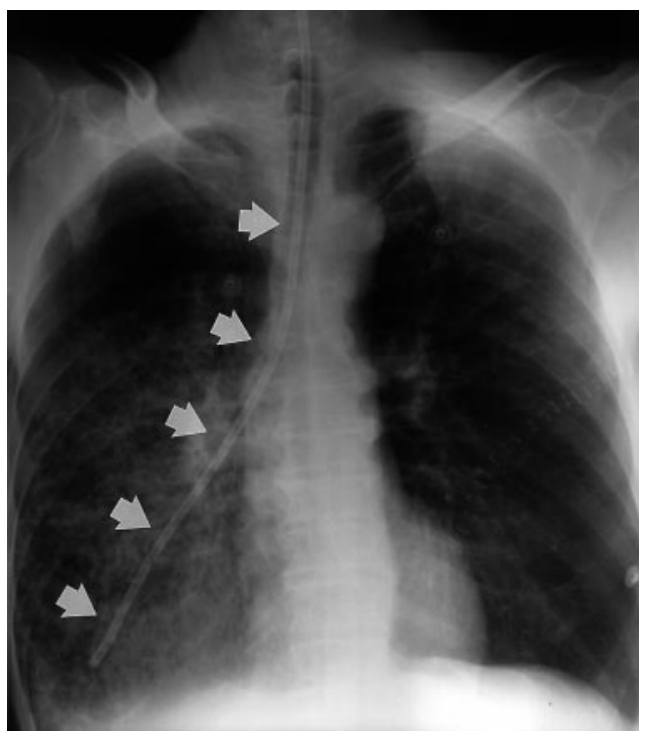

Abb. 84

Magensonden-Fehllage. Das Thoraxröntgen eines Lungentransplantierten zeigt die fehlpositionierte Magensonde (weiße Pfeile), die dem Verlauf der Trachea und des rechten Hauptbronchus folgt und mit ihrer Sitzlage weit distal im Tracheobronchialbaum zu liegen kommt. Solche Fehllagen können mit Bronchial-Perforationen und konsekutiven Pneumothoraces einhergehen 
Aspirationspneumonien

Meist im Mittellappen. Die Ausdehnung auf benachbarte Lungenlappen ist möglich.

Radiologische Diagnose einer Pneumonie bei beatmeten Patienten ist oft schwer.

Aerobronchogramme

Computer-Tomographie Mögliche Unterscheidung von Empyem und Abszess.

Verzerrte Luftbronchogramme

Kompression

Therapeutischer Zugang: Empyeme schnellstmöglich drainieren, Abszesse können z.T. mit Erreger-spezifische Antibiotika behandelt werden. ursacht. Nur in Ausnahmefällen können auch Pilz- oder Streptokokken-Infektionen solche Verdichtungen hervorrufen.

Miliare Verdichtungen, deren Knötchen uniforme Größen von ca. 2 bis $4 \mathrm{~mm}$ aufweisen, sind hauptsächlich durch M. tuberkulosis, in Ausnahmefällen auch durch Pilze verursacht. Fleckige Oberlappenverdichtungen lassen das Vorliegen von tuberkulösen oder fungalen Veränderungen vermuten. Im Intensivpatienten sind hier Histoplasmen und Kryptokokken die häufigsten Erreger. Ausgedehntere Pleuraergüsse sind häufig mit Pneumonien vergesellschaftet, die durch Anaerobier, Gram-negative Bakterien, S. aureus und S. pyogenes verursacht sind.

Bei Intensivpatienten liegt einer Pneumonie oft eine vorausgegangene Aspiration von Fremdmaterial zugrunde. Diese $>$ Aspirationspneumonien finden sich meist im Mittellappen bzw. in der Lingula und erscheinen in ihrem Anfangsstadium als parakardial gelegene peribronchiale streifige Verdichtungen. Kommt es zu einer Mitreaktion des umgebenden Lungenparenchyms, können die daraus entstehenden fleckigen Verdichtungen von Zeichen des Volumenverlustes begleitet sein. Die Aspirationspneumonie kann sich von ihrem fokalen Ursprungsort auf benachbarte Lungenareale ausdehnen.

Bei beatmeten Patienten fällt die radiologische Diagnose einer Pneumonie oft besonders schwer. Pneumonien, Ödeme, das ARDS, Infarkte und Blutungen können ähnliche Röntgenmuster aufweisen, sodaß die zuverlässige Bestätigung des Vorliegens einer Pneumonie aufgrund des Röntgenbildes allein oft nicht möglich ist. Das Röntgenmerkmal der Aerobronchogramme hat hier mit ca. $70 \%$ den besten prädiktiven Wert; dieses Zeichen ist jedoch auch für das ARDS relativ typisch. Um eventuellen Fehlinterpretationen pulmonaler Verdichtungen vorzubeugen, sollten pneumoniesuspekte Areale immer mit Vorbildern verglichen werden. Zudem sollte jede Änderung des Beatmungsregimes dem Radiologen mitgeteilt werden.

\section{Empyem, Abszess}

Sterile parapneumonische Pleuraergüsse verhalten sich radiologisch ähnlich wie andere primäre und sekundäre nicht-infektiöse Ergüsse; zwar können pleurale Narben zu Lokulationen führen, sonst aber dehnen sich Pleuraergüsse der Schwerkraft entsprechend aus. Demgegenüber zeigen fibropurulente Flüssigkeitsansammlungen eine starke Lokulationstendenz, sodaß auch bei Lageänderung des Patienten die Lage des Empyems gleichbleiben kann. Im Unterschied zu nicht-bettlägrigen Patienten, bei denen unkompliziert Röntgenaufnahmen in mehreren Ebenen angefertigt werden können, ist die Differenzierung zwischen Empyem und peripherem Lungenabszess im Intensivpatienten allein aufgrund der Röntgenaufnahme in einer Ebene extrem schwierig. Hier kann die CT von diagnostischer Bedeutung sein [9, 10]. Die Kriterien, welche zur Unterscheidung zwischen Empyem und Abszess herangezogen werden, sind: Form, Wandcharakteristika, umgebendes Gewebe.

Empyeme weisen meist eine linsenartige Konfiguration auf und bilden mit der Thoraxwand einen stumpfen Winkel. Demgegenüber erscheinen Abszesse sphärisch und bilden mit der Thoraxwand einen spitzen Winkel. Die Wand eines Empyems wird von verdickter Pleura gebildet. Die Dicke der Pleura ist ebenmäßig und weichteildicht. Demgegenüber hat der Abszess unregelmäßige Begrenzungen, die meist auch dicker sind als die Begrenzungen von Empyemen. Abszesswände können Lufteinschlüsse beinhalten und weisen manchmal verzerrte Luftbronchogramme auf.

Einem Empyem benachbarte Lungenabschnitte sind, insbesonders bei großen Empyemen, oftmals komprimiert. Diese Kompression kann zur Verlagerung von benachbarten Gefäßen und Bronchien führen. Da Abszesse benachbartes Lungengewebe eher zerstören als verdrängen, werden Gefäße und Bronchien hier nicht verlagert. Auch wenn die Unterscheidung zwischen Empyem und Abszess aufwendige Techniken wie die CT erfordert, sollte diese Differenzierung in Intensivpatienten immer angestrebt werden, da sie den therapeutischen Zugang grundlegend beeinflußt: während Empyeme schnellstmöglich drainiert werden sollten, können im Fall des Abszesses Erreger-spezifische Antibiotika den Bedarf nach einer invasiven Therapie hintanhalten. 


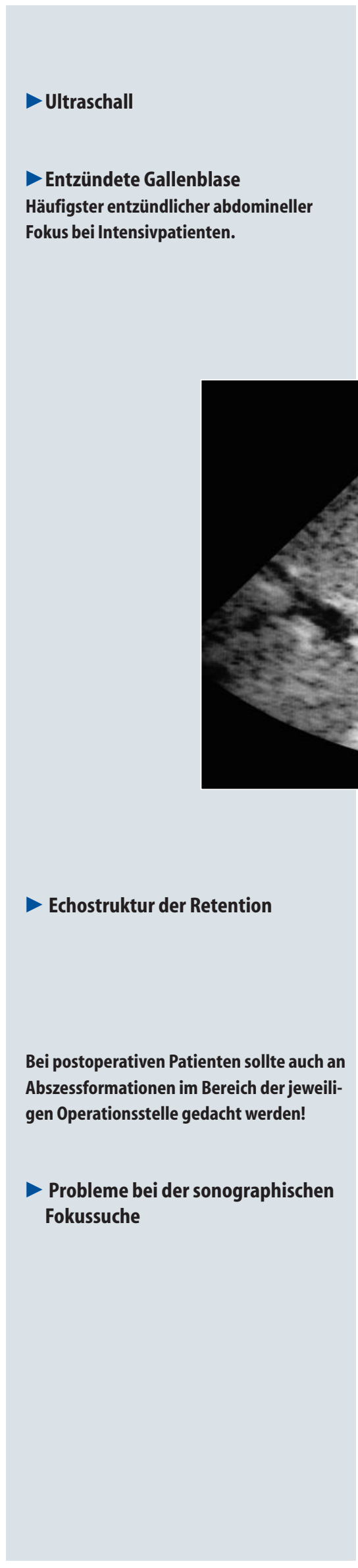

\section{Abdomineller Fokus: Bed-Side Diagnostik}

Die am Krankenbett einsetzbaren Techniken zur Suche nach einem abdominellen Fokus beschränken sich prinzipiell auf den Ultraschall. Hier muß die Aufmerksamkeit des Untersuchers in erster Linie pathologisch veränderten Organen und pathologischen Flüssigkeitsansammlungen dienen. Ein häufiger Fokus bei Intensivpatienten ist die entzündete Gallenblase. Sonographisch erscheint diese meist vergrößert, reichlich flüssigkeitsgefüllt, und mit verdickter dreigeschichteter Wand (echoreicher Außenstreifen, echoarmer Mittelstreifen, echoreicher Innenstreifen). Als Grenzwert für eine Vergrößerung gilt ein maximaler Durchmesser von ca. $8 \mathrm{~cm}$, als Wandverdickung gilt jeder Wanddurchmesser von über $4 \mathrm{~mm}$. Häufig zeigt sich im angrenzenden Leberparenchym ein echoarmer Randwall (Abb. 9). 


\section{Septierung von Retentionen}

\section{Röntgenologischen Lagekontrolle der Katheter}

\section{Radiologisch sichtbare Komplikationen:}

- Fehllage der Katheterspitze - Pneumothorax durch Verletzung

- Perforation zentraler Venen - Katheterbruch gen Abszesse typischerweise eine starke Anfärbung ihrer Membran, während ihr Zentrum dazu relativ hypodens bleibt; nicht-abgekapselte Retentionen zeigen naturgemäß keine Randanfärbung.

Im CT ist auch die eventuelle Septierung von Retentionen gut zu beurteilen, was wiederum von Bedeutung für eine einzuleitende Therapie ist. So können im Falle von septierten Retentionen unmittelbar nach der Diagnosesicherung durch das CT punktgenau Drainagen eingebracht werden, um eine optimale Entlastung zu gewähren. Zusammenfassend stellt die CT in der abdominellen Fokusdiagnostik den Goldstandard dar; sie sollte jedoch aufgrund des großen mit ihr verbundenen Aufwands nur in jenen Fällen zum Einsatz kommen, in denen die Sonographie keine konklusiven Ergebnisse gewährleistet.

\section{Radiologische Lagekontrolle intravaskulärer Zugänge}

\section{Zentralvenöse Katheter}

Die bevorzugt über die V. jugularis interna bzw. über die V. subclavia eingeführten Katheter sollten mit ihrer Spitze idealerweise in der V. cava superior zu liegen kommen und somit auf dem a.p.-Bild in Projektion auf den Bereich zwischen den sternalen Ansätzen der I. bis III. Rippe rechts gelangen. Bei der röntgenologischen Lagekontrolle dieser Katheter ist darauf zu achten, daß der Katheter immer in seinem gesamten intrathorakalen Verlauf dargestellt wird (also beispielsweise nicht mit abgeschnittener Spitze oder abgeschnittenem Ende). Sicherheitshalber sollte im Zuge der Erstkontrolle der Katheter mit Kontrastmittel dargestellt werden, um eine intravaskuläre Lage zweifelsfrei zu bestätigen.

Mögliche radiologisch sichtbare Komplikationen zentralvenöser Katheter sind Fehllagen der Katheterspitze (z.B. in der V.cava inferior, in der V.jugularis, in Venen der oberen Extremität), der Pneumothorax durch Verletzung der Pleura infolge der Venenpunktion, die Perforation zentraler Venen und der Katheterbruch mit konsekutiver Katheterembolisation (Abb. 10).
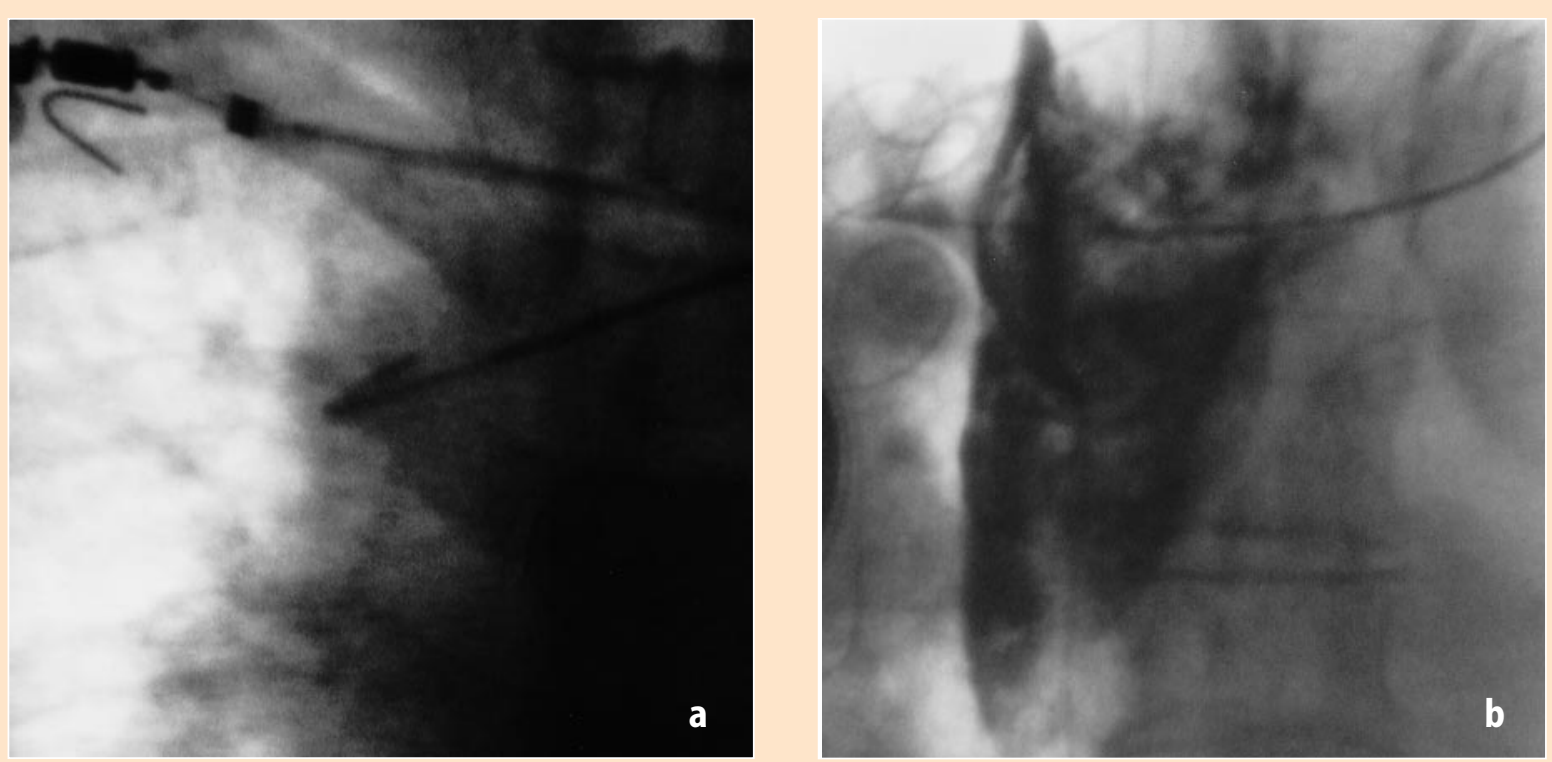

Abb. $10 \boldsymbol{\Delta}$

Detailaufnahme des oberen Mediastinum bei einem Patienten mit Fehllage eines zentralen Katheters in der Vena azygos vor (a) und nach (b) Ruptur des Gefäßes. Abb. 10a zeigt die nach oben und innen gerichtete Spitze des Katheters, die auf eine Fehllage in der Vena azygos deutet. Abb. 10b zeigt das flächige Kontrastmittelextravasat, das die Gefäßruptur bestätigt. 
Radiologische Kontrollen sollten auch nach erfolgloser zentralnervöser Punktion durchgeführt werden.

\section{Idealer Sitz der Katheterspitze}

\section{Komplikation des Swan-Ganz-Katheters}

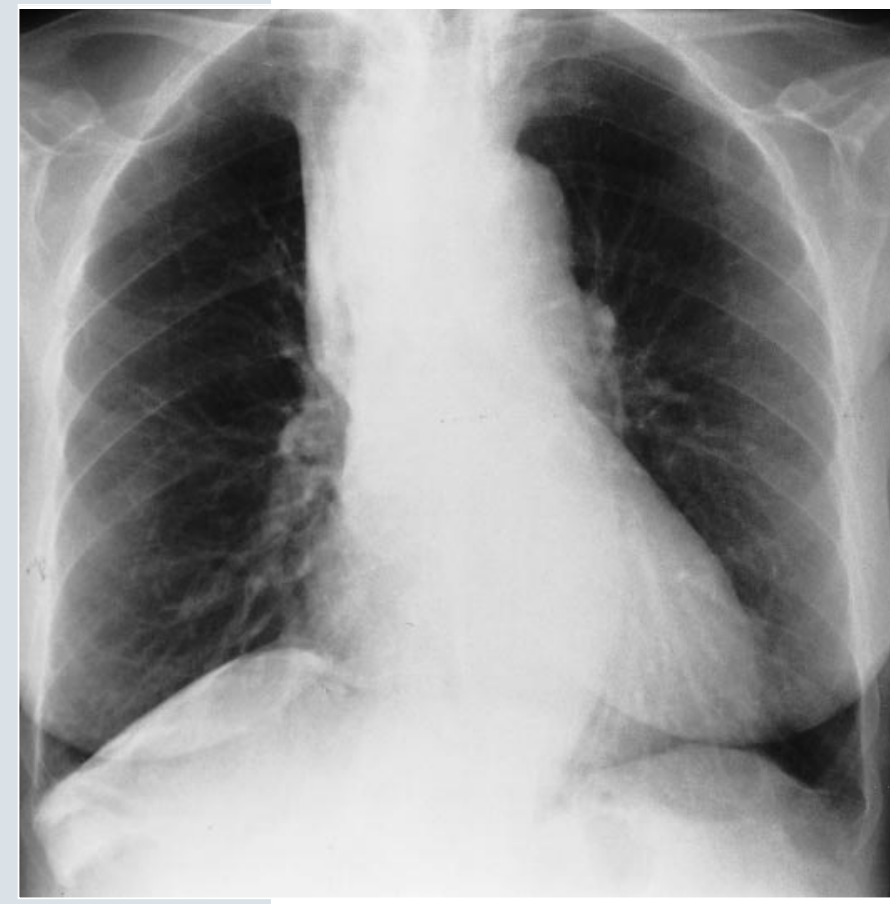

Lungenröntgen des gleichen Patienten wie in Abb. 10 zwei Tage nach Entlassung aus der Intensivstation. Noch immer zeigen sich rechts paramediastinal und rechts pleural Kontrastmittelreste
Es bleibt anzumerken, daß radiologische Kontrollen auch nach erfolgloser zentralvenöser Punktion durchgeführt werden sollten, um Komplikationen wie Pneumothorax oder größere Weichteilhämatome auszuschließen. Liegen mehrere Katheter in einem Gefäß (z.B.V. cava oder V. subclavia) vor, ist häufig zu beobachten, daß sich diese Katheter in ihrem Verlauf überkreuzen. Liegt dieses Überkreuzen nicht vor, sollte an die Möglichkeit einer intraarteriellen Fehllage eines der Katheter gedacht werden $[14,16]$.

\section{Swan-Ganz Katheter}

Der ideale Sitz der Katheterspitze ist der rechte oder linke Hauptstamm der Pulmonalarterie. Im Regelfall genügt die a.p.-Aufnahme, um eine suffiziente Katheterlage zu bestätigen, doch kann im Zweifelsfall das Anfertigen eines Seitbildes angestrebt werden (Abb. 11). Abgesehen von Schlingenbildungen im rechten Vorhof, welche auf dem a.p.-Bild gut zur Darstellung kommen, stellt die schwerwiegendste - Komplikation des Swan-Ganz-Katheters, nämlich die Verlegung einer Pulmonalarterie durch einen nicht desoufflierten Ballon bzw. durch eine gebrochene Katheterspitze, eine Indikation zur Computertomographie dar, da hier das genaue Ausmaß eventueller pulmonaler Parenchymschädigung beurteilt werden kann.
Proximale Katheter-Fehllage

\section{Intraaortale Ballonpumpe}

Während der Diastole imponiert die Pumpe als längliche, gasgefüllte Struktur, die dem topographischen Verlauf der Aorta folgt. Während der Systole ist der desoufflierte Ballon nicht sichtbar; lediglich ein röntgendichter Marker, der die Spitze des Katheters bezeichnet, läßt sich darstellen. Idealerweise liegt die Katheterspitze genau distal der linken A. subclavia und kommt demnach auf dem a.p.-Bild in Projektion auf den Aortenbogen zur Darstellung.

$\mathrm{Zu}$ den möglichen radiologisch erkennbaren Komplikationen zählen die proximale Katheter-Fehllage mit konsekutiver Obstruktion der linken A. subclavia bzw. eventuell hirnversorgender Gefäße, die distale Katheter-Fehllage mit konsekutivem Funktionsdefizit der Pumpe, die Dissektion der Aortenwand, sowie die Ballon-Ruptur mit konsekutiver Gasembolie. Die regelrechte Lage der intraaortalen 
Ballonpumpe läßt sich nativradiologisch gut überprüfen. Aufgrund der tiefgreifenden Konsequenzen möglicher Komplikationen sollte jedoch nicht gezögert werden, zur weiteren Abklärung die Computertomographie einzusetzen. Von der Aortographie sollte abgesehen werden, da die meisten Träger intraaortaler Ballonpumpen heparinisiert sind.

Teil II des Beitrags erscheint in der Dezember-Ausgabe von "DER RADIOLOGE" (Band 38, Heft 12, 1998)

\section{Literatur}

1. Angstwurm H, Bachmann KD, Böckle $F$ et al. (1991) Kriterien des Hirntodes; Entscheidungshilfen zur Feststellung des Hirntodes. Dt Ärztebl 88:80-91

2. Bankier AA, Wiesmayr MH, Henk C, Turetschek K, Winkelbauer G, Mallek R, Fleischmann D, Sanata K, Herold C (1997) Radiographic detection of intrabronchial malpositions of masogastric tubes and subsequent complications in intensive care unit patients. Intensive Care Medicine 23:406-410

3. Bankier AA, Mallek R, Wiesmayr MM, Fleischmann D, Kranz A, Kontrus M, Knapp S, Winkelbauer F (1997) Azygos arch cannulation by central venous catheters: Radiographic detection of malposition and subsequent complications.Journal of Thoracic Imaging 12:64-69

4. Chiles C, Ravin CE (1986) Radiographic recognition of pneumothorax in the intensive care unit. Crit Care Med 14:677-680

3. Gattinoni L, Pelosi P, Pesenti A, Brazzi L,Vitale G, Moretto A, Crespi A, Tagliabue M (1991) CT scan in ARDS: clinical and physiological insights. Acta Anaesthesiol Scand 95:87-96

4. Görzer H., Heimberger K., Schindler E (1994) Spiral CT-Angiography with digital subtraction. J Comput Assist Tomogr 18:839-841

5. Gray P, Sullivan G, Ostryzniuk P, McEwen TAJ, Rigby M, Roberts DE (1992) Value of postprocedural chest radiographs in the adult intensive care unit. Crit Care Med 20:1513-1518

6. Hunt WE, Hess RM (1968) Surgical risk as related to time of intervention in the repair of intracranial aneurysms.J Neurosurg 28:14-19

7. Kopetzki C (1988) Organgewinnung zu Zwecken der Transplantation. In: Forschungen aus Staat und Recht, Band 82. Springer, Wien New York

8. Milne EN, Pistolesi M, Miniati M, Giuntini C (1985) The radiographic distinction of cardiogenic and noncardiogenic edema. Am J Roentg 144:879-884

9. Mirvis SE, Rodriguez A, Whitley NO, Tarr RJ (1985) CT evaluation of thoracic infections after major trauma. Am J Roentg 144:1183-1187

10. Mirvis SE, Tobin KD, Kostrubiak I, Belzberg H (1987) Thoracic CT in detecting occult diseases in critically ill patients. Am J Roentg 148:685-689

11. Peters PE, Bongartz G., Drews C (1990) Magnetresonanzangiographie der hirnversorgenden Arterien. Fortschr Röntgenstr 152:528-533

12. Reutern GMV (1991) Zerebraler Zirkulationsstillstand. Diagnostik mit der Dopplersonographie.Dt Ärzteblatt 88:55-65

13. Stark DD, Federle MP, Goodman PC (1983) CT and radiographic assessment of tube thoracostomy. Am J Roentg 141:253-258

14. Thompson MJ, Kubicka RA, Smith C (1989) Evaluation of cardiopulmonary devices on chest radiographs: digital versus analog radiographs. Am J Roentg 153:1165-1168

15. Toccino IM, Miller MH, Fairfax WR. Distribution of pneumothorax in the supine and semirecumbent critically ill adult. Am J Roentg 144:901-905

16. Wechsler RJ, Steiner RM, Kinori I (1988) Monitoring the monitors: the radiology of thoracic catheters, wires, and tubes. Sem Roentg 23:61-84

17. Woodring JH (1983) Recognition of pleural effusion on supine radiographs: how much fluid is required? Am J Roentg 142:59-64

18. Yu CJ, Yang PC, Chang DB, Luh KT (1992) Diagnostic and therapeutic use of chest sonography: value in critically ill patients. Am J Roentg 159:695-701

Der vorliegende Beitrag ist in ähnlicher Form bereits in einer anderen Springer-Facharztzeitschrift erschienen (Quelle:Der Anaesthesist 8/96, S.769-786). Für DER RADIOLOGE wurde der Beitrag überarbeitet, aktualisiert und von den Autoren für dieses Heft imprimiert. 